\title{
Clinical experience with venetoclax in patients with newly diagnosed, relapsed, or refractory acute myeloid leukemia
}

\author{
Maximilian Fleischmann ${ }^{1} \cdot$ Sebastian Scholl ${ }^{1}$ Jochen J. Frietsch ${ }^{1} \cdot$ Inken Hilgendorf ${ }^{1} \cdot$ Karin Schrenk $^{1}$. \\ Jakob Hammersen ${ }^{1} \cdot$ Florian Prims $^{2}$. Christian Thiede ${ }^{3}$. Andreas Hochhaus ${ }^{1}$. Ulf Schnetzke ${ }^{1}$ (i)
}

Received: 28 October 2021 / Accepted: 20 January 2022 / Published online: 31 January 2022

(c) The Author(s) 2022

\begin{abstract}
Background Diagnosis of acute myeloid leukemia (AML) is associated with poor outcome in elderly and unfit patients. Recently, approval of the $B C L-2$ inhibitor venetoclax (VEN) in combination with hypo-methylating agents (HMA) led to a significant improvement of response rates and survival. Further, application in the relapsed or refractory ( $\mathrm{r} / \mathrm{r}$ ) AML setting or in context of allogeneic stem cell transplantation (alloHSCT) seems feasible.

Methods and patients Fifty-six consecutive adult AML patients on VEN from January 2019 to June 2021 were analyzed retrospectively. Patients received VEN either as first-line treatment, as subsequent therapy ( $\mathrm{r} / \mathrm{r}$ AML excluding prior alloHSCT), or at relapse after alloHSCT. VEN was administered orally in 28-day cycles either combined with HMA or low-dose cytarabine (LDAC).

Results After a median follow-up of 11.5 (range 6.1-22.3) months, median overall survival (OS) from start of VEN treatment was 13.3 (2.2-20.5) months, $5.0(0.8-24.3)$ months and $4.0(1.5-22.1)$ months for first-line, subsequent line treatment and at relapse post-alloHSCT, respectively. Median OS was 11.5 (10-22.3) months from start of VEN when subsequent alloHSCT was carried out. Relapse-free survival (RFS) for the total cohort was $10.2(2.2-24.3)$ months. Overall response rate (composite complete remission + partial remission) was $51.8 \%$ for the total cohort $(61.1 \%$ for VEN first-line treatment, $52.2 \%$ for subsequent line and $42.8 \%$ at relapse post-alloHSCT). Subgroup analysis revealed a significantly reduced median OS in FLT3-ITD mutated AML with 3.4 (1.9-4.9) months versus 10.4 (0.8-24.3) months for non-mutated cases, (HR 4.45, 95\% CI 0.89-22.13, $p=0.0002$ ). Patients harboring NPMI or IDH1/2 mutations lacking co-occurrence of FLT3-ITD showed a survival advantage over patients without those mutations (11.2 (5-24.3) months versus $5.0(0.8-22.1)$ months, respectively, (HR $0.53,95 \%$ CI $0.23-1.21, p=0.131$ ). Multivariate analysis revealed mutated NPM1 as a significant prognostic variable for achieving complete remission (CR) (HR 19.14, 95\% CI $2.30-436.2, p<0.05$ ). The most common adverse events were hematological, with grade 3 and 4 neutropenia and thrombocytopenia reported in $44.6 \%$ and $14.5 \%$ of patients, respectively. Conclusion Detailed analyses on efficacy for common clinical scenarios, such as first-line treatment, subsequent therapy ( $\mathrm{r} / \mathrm{r}$ AML), and application prior to and post-alloHSCT, are presented. The findings suggest VEN treatment combinations efficacious not only in first-line setting but also in $\mathrm{r} / \mathrm{r}$ AML. Furthermore, VEN might play a role in a subgroup of patients with failure to conventional chemotherapy as a salvage regimen aiming for potential curative alloHSCT.
\end{abstract}

Keywords AML $\cdot$ Venetoclax $\cdot$ Hypo-methylating agents $\cdot$ Refractory $\cdot$ Relapse $\cdot$ Salvage therapy

Ulf Schnetzke

ulf.schnetzke@med.uni-jena.de

1 Klinik Für Innere Medizin II, Abteilung Für Hämatologie Und Internistische Onkologie, Universitätsklinikum Jena, Am Klinikum 1, 07747 Jena, Germany

2 Klinik Für Innere Medizin, Abteilung Für Hämatologie Und Onkologie, SRH Klinikum Burgenlandkreis Naumburg, Naumburg, Germany

3 Medizinische Klinik I, Universitätsklinikum Carl Gustav Carus, Dresden, Germany

\section{Introduction}

Acute myeloid leukemia (AML) in elderly unfit patients remains challenging and overall long-term prognosis is poor (Juliusson et al. 2009). New treatment options especially for frontline treatment have emerged over the past years. First, introduction of hypo-methylating agents (HMAs) replaced conventional chemotherapy as first-line therapeutic option in this patient population (Dombret 
et al. 2015; Kantarjian et al. 2012). More recently, the B-cell leukemia/lymphoma-2 (BCL-2) inhibitor venetoclax (VEN) has been approved and led to a noteworthy impact on disease management (Apel et al. 2021; Samra et al. 2020; Pollyea et al. 2019; Kayser and Levis 2021).

Two recently published phase 3 studies demonstrated an overall survival (OS) benefit for the combination of HMA or low-dose cytarabine (LDAC) with VEN compared to single agent therapy (DiNardo et al. 2020; Wei et al. 2020).

The VIALE-A trial (NCT02993523) included 431 AML patients (286 azacitidine (AZA) plus VEN and 154 with AZA alone) with a median age of 76 years (DiNardo et al. 2020). The median OS was improved from 9.6 (7.4 - 18.7) months to 14.7 (11.9 - 18.7; HR 0.66) months with the VEN combination therapy. Attaining complete remission was more likely with AZA-VEN than with the control regime (36.7\% versus $17.9 \%$ ). Of note, improvement of response rates was seen across all genomic risk groups including adverse cytogenetic risk and high-risk molecular mutations.

Within another large phase 3 trial, 210 patients were treated either with LDAC alone or VEN and LDAC (Wei et al. 2020). The median OS was 4.1 months, as compared to 7.2 months within the combination arm. Although this VIALE-C trial (NCT03069352) did not meet its primary survival endpoint, the data show a potential survival benefit of the combination of VEN and LDAC and improved response rates.

Therefore, HMA-VEN combination received full U.S. Food and Drug Administration (FDA) approval in October 2020 and in May 2021 by the European Medicines Agency (EMA) as first-line therapy for patients not eligible for intensive treatment. LDAC-VEN combination has exclusively been approved by the FDA yet.

Due to improved response rates and excellent tolerability, there are ongoing debates as to whether VEN combination strategies should be implemented into first-line treatment for subsets of newly diagnosed AML patients treating in a curative intent (DiNardo et al. 2021; Maiti et al. 2021).

Compared to frontline treatment, limited data of HMA-VEN combination exist on its effect in the relapsed/ refractory $(r / r)$ setting. Encouraging results have been reported in small retrospective studies evaluating outcomes of $r / r$ AML patients treated with VEN as single agent or in combination with other conventional agents (DiNardo et al. 2018; Aldoss et al. 2018; Piccini et al. 2021).

Here, we sought to analyze efficacy and tolerability of VEN therapy at an academic site, both in treatment-naïve and $r / r$ AML patients. A special focus was set on common clinical settings, such as VEN application in case of induction treatment failure or at relapse following allogeneic stem cell transplantation (alloHSCT).

\section{Patients and methods}

\section{Patient cohort}

A total of 56 consecutive adult patients receiving VEN for AML treatment from January 2019 to June 2021 were analyzed. VEN was applied as (i) first-line therapy $(n=18)$, (ii) subsequent line (r/r AML including salvage following failure of conventional induction chemotherapy and excluding patients with prior alloHSCT) $(n=23)$, and (iii) at relapse post-alloHSCT $(n=15) .27$ patients $(48.2 \%)$ had de novo, $28(51.8 \%)$ secondary AML (sAML) derived from myeloproliferative diseases (MPN) or myelodysplastic syndromes (MDS) and one patient (1.8\%) treatment-associated AML. Treatment response was adjudicated according to European LeukemiaNet (ELN) 2017 recommendations (Döhner et al. 2017) (Table 1).

\section{Informed consent}

All patients gave their written informed consent for data acquisition and analysis. All patients were included in the SAL (Study Alliance Leukemia) registry. The analysis was approved by local ethics committee of the University Hospital Jena, Germany (no. 3967-2/13 for SAL registry).

\section{Patients' treatment}

VEN was administered orally using a 28-day cycle either combined with HMA (decitabine $20 \mathrm{mg} / \mathrm{m}^{2}$ intravenously daily on days $1-5$, 5 -azacitidine $75 \mathrm{mg} / \mathrm{m}^{2}$ subcutaneously for 7 consecutive days, repeated after 28 days each) or lowdose cytarabine (LDAC $40 \mathrm{mg}$ subcutaneously on days 1-7, repeated after 28 days) (Jonas and Pollyea 2019). Dose adjustment of VEN was required depending on concomitant azole therapy, tolerance and cytopenia (Jonas and Pollyea 2019).

The majority of patients undergoing alloHSCT received a reduced-toxicity conditioning (RTC) based on treosulfan or busulfan in combination with fludarabine with or without ATG prior to transplantation $(n=18,81.8 \%)$ (Casper et al. 2012; Beelen et al. 2020). The remaining patients underwent myeloablative conditioning (MAC) $(n=4,18.2 \%)$ (Jethava et al. 2017). Further characteristics of patients undergoing alloHSCT are summarized in Table S1.

\section{Safety analyses}

Hematologic and non-hematologic toxicity was evaluated according to the Common Terminology Criteria and Adverse Events classification (CTCAE v5.0). For classification of 
Table 1 Patient demographics

\begin{tabular}{|c|c|}
\hline & $n=56$ \\
\hline Sex, female $(\%)$ & $24(42.8)$ \\
\hline Median age, years (range) & $66.5(34-83)$ \\
\hline \multicolumn{2}{|c|}{ ECOG performance status, $n(\%)$} \\
\hline $0-1$ & $31(55.4)$ \\
\hline $2-3$ & $25(44.6)$ \\
\hline \multicolumn{2}{|l|}{ FAB classification, $n(\%)$} \\
\hline M0 & $0(0)$ \\
\hline M1/M2 & $15(26.7)$ \\
\hline M3 & Excluded \\
\hline $\mathrm{M} 4 / 5$ & $20(35.7)$ \\
\hline M6 & $2(3.5)$ \\
\hline M7 & $1(1.8)$ \\
\hline n.a & $18(32.1)$ \\
\hline \multicolumn{2}{|l|}{ AML type, $n(\%)$} \\
\hline de novo & $27(48.2)$ \\
\hline s-AML & $28(51.8)$ \\
\hline $\mathrm{t}-\mathrm{AML}$ & $1(1.8)$ \\
\hline \multicolumn{2}{|c|}{ Blood count at baseline, median in $10^{9} / 1$ (range) } \\
\hline WBC & $12(0-317)$ \\
\hline $\mathrm{RBC}$ & $4.5(4.8-8)$ \\
\hline ANC & $0.39(0-12.5)$ \\
\hline \multicolumn{2}{|c|}{ Cytogenetic abnormalities, $n(\%)$} \\
\hline Normal karyotype & $18(32.1)$ \\
\hline Complex aberrant & $15(26.7)$ \\
\hline Monosomy 7 & $2(3.5)$ \\
\hline Trisomy 8 & $6(10.7)$ \\
\hline Inversion 3 & $3(5.3)$ \\
\hline $5 q-$ & $2(3.6)$ \\
\hline Other & $9(16)$ \\
\hline n.a & $1(1.8)$ \\
\hline \multicolumn{2}{|l|}{ Cytogenetic risk, $n(\%)$} \\
\hline Favorable & $0(0)$ \\
\hline Intermediate & $34(60.7)$ \\
\hline Adverse & $21(37.5)$ \\
\hline n.a & $1(1.8)$ \\
\hline \multicolumn{2}{|c|}{ ELN risk stratification $2017, n(\%)$} \\
\hline Favorable & $5(8.9)$ \\
\hline Intermediate & $17(30.3)$ \\
\hline Adverse & $32(57.1)$ \\
\hline n.a & $2(3.6)$ \\
\hline \multicolumn{2}{|c|}{ Molecular genetics at start VEN, $n(\%)$} \\
\hline No aberrations detected & $19(33.9)$ \\
\hline MLL rearrangement & $3(5.3)$ \\
\hline KMT2A-PTD & $5(8.9)$ \\
\hline FLT3-ITD mut & $8(14.3)$ \\
\hline FLT3-TKD mut & $4(7.1)$ \\
\hline NPM1 mut & $9(16)$ \\
\hline IDH1/2 mut & $7(12.5)$ \\
\hline TP53 mut & $5(8.9)$ \\
\hline PTPN11 mut & $2(3.6)$ \\
\hline RUNX1 mut & $5(8.9)$ \\
\hline Missing data & $2(3.6)$ \\
\hline
\end{tabular}

Table 1 (continued)

ECOG Eastern cooperative oncology group, FAB French- AmericanBritish, $s-A M L$ secondary acute myeloid leukemia, $t-A M L$ treatmentrelated AML, AlloHSCT allogeneic hematopoietic stem cell transplantation, n.a. not assessed, $E L N$ European LeukemiaNet, $R B C$ red blood count, $W B C$ white blood count, $A N C$ absolute neutrophil count

hematological toxicity changes for neutrophils, platelets and hemoglobin compared to baseline values at start of VEN treatment have been assessed.

\section{Cytogenetic and molecular genetic analysis}

Cytogenetic evaluation was performed using standard banding techniques, and karyotypes were described according to the currently valid International System for Human Cytogenetic Nomenclature (McGowan-Jordan et al. 2021). Cytogenetic categorization into favorable, intermediate or adverse risk was performed on the basis of recommended criteria (Döhner et al. 2017). Detections of AML-specific molecular aberrations according to ELN 2017 guidelines were performed by next-generation sequencing (NGS) as published previously (Stasik et al. 2020). Genetic characteristics before initiating VEN treatment have been assessed and reported in Table 1.

\section{Response assessment}

Response assessment was carried out separately for three disease settings: first-line, subsequent line ( $\mathrm{r} / \mathrm{r}$ AML excluding patients who underwent prior alloHSCT) and relapse post-alloHSCT. Subgroup analyses were accomplished for patients achieving composite complete remission (CRc) and for distinct molecular aberrations (FLT3-ITD, NPMI, and IDH1/2).

Efficacy assessments were performed by calculation for OS, progression-free survival (PFS) and survival from start VEN treatment to last follow-up or death from any cause. OS is defined as date of first diagnosis of AML to date of last follow-up and death from any cause. PFS is defined as date of initiation of VEN until progression/relapse or last followup/death from any cause. Relapse-free survival (RFS) was calculated for patients attaining $\mathrm{CRc}$ measured from date of remission to relapse or last follow-up.

Overall response rate (ORR) was defined as CRc comprising complete remission, complete remission with incomplete hematological recovery (CRi) and complete remission with incomplete platelet recovery (CRp) and partial remission (PR). Additional response criteria were applied according to ELN 2017 guidelines (Döhner et al. 2017). CR was defined as $5 \%$ blasts or less within the bone marrow and adequate peripheral blood counts (neutrophils $\geq 1.0 \times 10^{9} / 1$, platelets $\left.\geq 100 \times 10^{9} / \mathrm{l}\right)$. Partial remission was defined as $5-25 \%$ 
blasts in the bone marrow and a total reduction of blasts of at least $50 \%$ of AML blasts. Progressive disease (PD) was defined as increase of bone marrow and/or peripheral blast count or new extramedullary manifestations. Stable disease (SD) was defined when CRc, PR or PD are not met and last for at least 3 months.

Additionally, transfusion requirements were also assessed for platelets and red blood cells (RBC). Transfusion dependence was determined by Gale criteria with greater than or equal to 2 units per month over the prior 3 months (Gale et al. 2011).

\section{Statistics}

Time-to-event analyses (OS, PFS) were estimated using Kaplan-Meier method and compared using log-rank test. $p$ values of $<0.05$ were considered as statistically significant. Statistical analyses were performed using GraphPad Prism 8.0.2 (GraphPad Inc., San Diego, CA, USA).

\section{Results}

\section{Baseline characteristics}

A total of 56 adult AML patients with a median age of 66.5 (range 34-83) years were included in this analysis. Table 1 provides information about morphology, AML-type and genetics including risk stratification.

48.2\% ( $n=27)$ presented with de novo AML, 51.8\% $(n=29)$ with secondary AML (sAML) including one patient with treatment association (Table 1). The median time of myelodysplastic syndrome (MDS) diagnosis to
sAML development was 11.7 (1.7 - 156.4) months (data not shown).

Eastern Cooperative Oncology Group (ECOG) performance status rated 31 patients $(55.3 \%)$ as $0-1$ and 25 patients (44.6\%) as $2-3$, respectively.

Cytogenetic risk profiling was performed for 55 of 56 patients $(98.2 \%)$ and resulted in favorable, intermediate and adverse risk in $0(0 \%), 34(60.7 \%)$ and 21 (37.5\%), respectively. Data for molecular aberrations were available in 54 of $56(96.4 \%)$ AML patients. Risk stratification according to the current ELN criteria 2017 showed a distribution in favorable, intermediate and adverse risk in 5 (8.9\%), 17 $(30.3 \%)$ and $32(57.1 \%)$ of patients, respectively (Table 1$)$. Further characteristics of those patients who received alloHSCT $(39.3 \%, n=22)$ are summarized in Table $\mathrm{S} 1$.

\section{Treatment characteristics}

Treatment intentions are illustrated in the CONSORT diagram (Fig. 1) and detailed information is provided in Table 2.

For first-line therapy, VEN was applied in $18 \mathrm{AML}$ patients, 23 patients received $\mathrm{VEN}$ as subsequent therapy (r/r AML without prior alloHSCT). A third subgroup was treated with VEN due to disease relapse following alloHSCT $(n=15)$. Of note, 7 patients were successfully transitioned to alloHSCT after VEN treatment (demographics and survival are reported in Table $\mathrm{S} 1$ and Figure S1, C, respectively). Notably, one patient received VEN/HMA as first-line treatment before subsequent alloHSCT. Since all other VEN/ HMA first-line patients $(n=17)$ were treated within a palliative setting, the only patient underwent alloHSCT was not

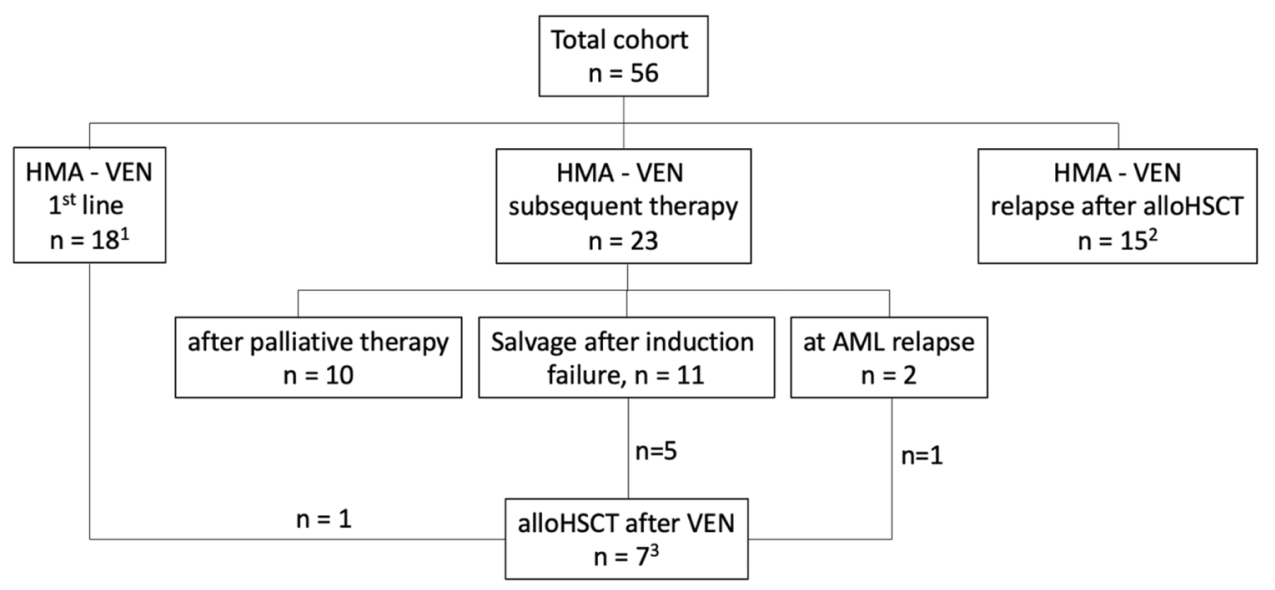

Fig. 1 CONSORT diagram: detailed overview about different treatment cohorts and disease settings. ${ }^{1}$ One pt received HMA-VEN as first-line treatment with intention to alloHSCT. ${ }^{2}$ Including 2 pts with AML relapse after second alloHSCT. ${ }^{3} 3$ pts received VEN twice in relapse after alloHSCT without response (excluded from analysis). $V E N$ Venetoclax, $p t(s)$ patient(s), HMA hypo-methylating agent, alloHSCT allogeneic hematopoietic stem cell transplantation 
Table 2 Treatment characteristics

\begin{tabular}{|c|c|}
\hline \multicolumn{2}{|l|}{ Treatment cohorts, $n(\%)$} \\
\hline VEN first-line, palliative & $17(30.4)$ \\
\hline Median age & $77(54-83)$ \\
\hline VEN $>1$ line, palliative & $17(30.4)$ \\
\hline Median age & $71(50-81)$ \\
\hline VEN at relapse post-alloHSCT & $15(26.7)$ \\
\hline Median age & $56(34-65)$ \\
\hline VEN as salvage prior to alloHSCT & $7(12.5)$ \\
\hline Median age & $61(50-72)$ \\
\hline \multicolumn{2}{|l|}{ Remission status prior to VEN, $n(\%)$} \\
\hline Progressive disease & $50(89.2)$ \\
\hline Partial remission & $4(7.1)$ \\
\hline Complete remission & $1(1.8)$ \\
\hline Stable disease & $1(1.8)$ \\
\hline Refractory to any line prior VEN & $22 / 38(57.9)$ \\
\hline Lines prior to VEN, median (range) & $1.5(0-8)$ \\
\hline \multicolumn{2}{|l|}{ Combination with VEN, $n(\%)$} \\
\hline Decitabine & $22(39.3)$ \\
\hline 5-Azacitidine & $28(50)$ \\
\hline LDAC & $2(3.6)$ \\
\hline More than one (VEN beyond progression)* & $4(7.1)$ \\
\hline Cycles VEN applied, median (range) & $3(1-18)$ \\
\hline \multicolumn{2}{|l|}{ Dosing } \\
\hline Months of VEN treatment, median (range) & $3.4(0.6-22.1)$ \\
\hline Total amount applied in mg, median (range) & $13,920(640-106,720)$ \\
\hline Mean dosage of VEN in mg (range) & $149(20.9-362,5)$ \\
\hline Concurrent azole application, $n(\%)$ & $48 / 56(85.7)$ \\
\hline Posaconazole & $23(47.9)$ \\
\hline Fluconazole & $11(22.9)$ \\
\hline Isavuconazole & $9(18.7)$ \\
\hline More than one azole (consecutively) & $5(10.4)$ \\
\hline VEN interruption, $n(\%)$ & $16(28.6)$ \\
\hline Due to neutropenia & $15(93.7)$ \\
\hline Due to nausea & $1(6.2)$ \\
\hline CR achieved before VEN, median (range) & $1(0-4)$ \\
\hline PR achieved before VEN, median (range) & $0.5(0-4)$ \\
\hline \multicolumn{2}{|l|}{ Prior treatment lines before VEN, $n$} \\
\hline HMA & 22 \\
\hline LDAC & 8 \\
\hline Consolidation chemotherapy & 14 \\
\hline Induction/re-induction & 44 \\
\hline alloHSCT & 15 \\
\hline TKI & 6 \\
\hline Other & 9 \\
\hline Treatment lines post VEN, $n$ & $18 / 56$ \\
\hline alloHSCT & 7 \\
\hline Quizartinib & 1 \\
\hline LDAC & 7 \\
\hline Second VEN application & 2 \\
\hline Clinical trial & 1 \\
\hline
\end{tabular}

VEN venetoclax, alloHSCT allogeneic stem cell transplantation, $L D A C$ low-dose cytarabine, HMA hypo-methylating agents, $C R$ complete remission, $P R$ partial remission, $T K I$ tyrosine kinase inhibitor

*VEN was continued beyond progression while the backbone (HMA/ LDAC) was changed included in survival analysis for first-line VEN treatment group.

The majority of patients received VEN in combination with an HMA agent backbone, including 22 (39.3\%) decitabine and 28 (50\%) AZA (Table 2).

Median time of VEN treatment was $3.4(0.6-22.1)$ months with a median application of 3 (1-18) treatment cycles (28 days). The overall mean drug dosage was 149 (20.9-362.5) mg. In 85.7\% of all patients, concurrent azole medication was applied (posaconazole $47.9 \%$, fluconazole $22.9 \%$ and isavuconazole $18.7 \%$ ). Interruption of treatment was necessary in 16 patients (28.6\%) being the majority due to grade 4 neutropenia (93.7\%) and one patient due to severe nausea and emesis $(6.2 \%)$.

22 of 38 patients $(57.9 \%)$ were refractory to any treatment line prior to VEN. In median, one CR (range 0-4) with a median of 1.5 (range $0-8$ ) therapy lines could be achieved prior to initiation of VEN treatment. Information on prior or subsequent treatment regimens to VEN is indicated in Table 2. Importantly, except for 3 patients receiving alloHSCT, no response to further treatment approaches after VEN could be achieved.

\section{Response to VEN treatment}

Subgroup analyses with respect to treatment intention showed a median OS starting at VEN treatment initiation of 13.3 (2.2-20.5) months, 5.0 (0.8-24.3) months, 4.0 (1.5-22.1) months for first-line treatment, subsequent line treatment and post-alloHSCT, respectively (Fig. 2A, B). Median follow-up was $11.5(6.1-22.3)$ months since diagnosis of AML. Of note, survival rates for patients who received VEN prior to alloHSCT were calculated separately with a median OS of 11.5 (10.4-22.3) months (Figure S1, C).

Patients who achieved CRc on VEN treatment had a median OS of 20.8 (2.5-24.3) months and PFS of 20.5 (2.6 - 24.3) months, respectively (Fig. 2C, D).

For the whole cohort, OS starting at AML diagnosis was $18.7(2.8$ - 125.3) months and not reached in patients who received CRc (2-year OS: 63\%) (Figure S1 A, B). RFS for the whole cohort was $10.2(2.2-24.3)$ months (Figure S1, D).

An ORR of 51.8\% was achieved for the whole population (data not shown). In detail, ORR of $61.1 \%$ was seen when VEN was applied as first-line treatment, $52.2 \%$ for subsequent line treatment and $42.8 \%$ at relapse post-alloHSCT (Fig. 3). Median time to first response for patients achieving CRc was $2.7(1.4-16.2)$ months. Differences in ORR were also seen when comparing distinct genetic subgroups. Patients with cytogenetically intermediate-risk features showed an ORR of $57.5 \%$ whereas only $42.8 \%$ in high-risk patients (Fig. 3B). Presence of FLT3-ITD was associated 

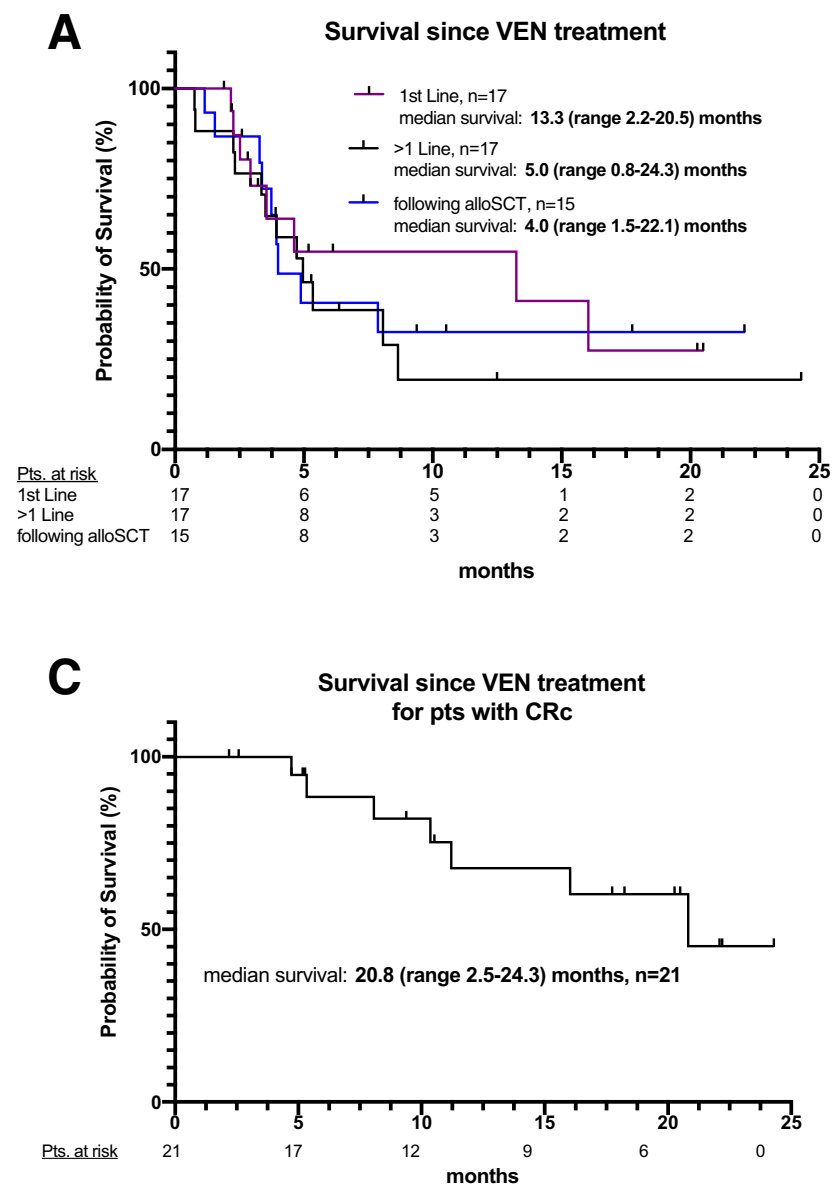

Fig. 2 Kaplan-Meier estimates for OS and PFS were measured from starting VEN treatment in indicated groups (A and $\mathbf{B}$, respectively), OS and PFS since VEN initiation in patients who achieved $\mathrm{CRc}$ (C and D, respectively). OS overall survival, alloHSCT alloge-
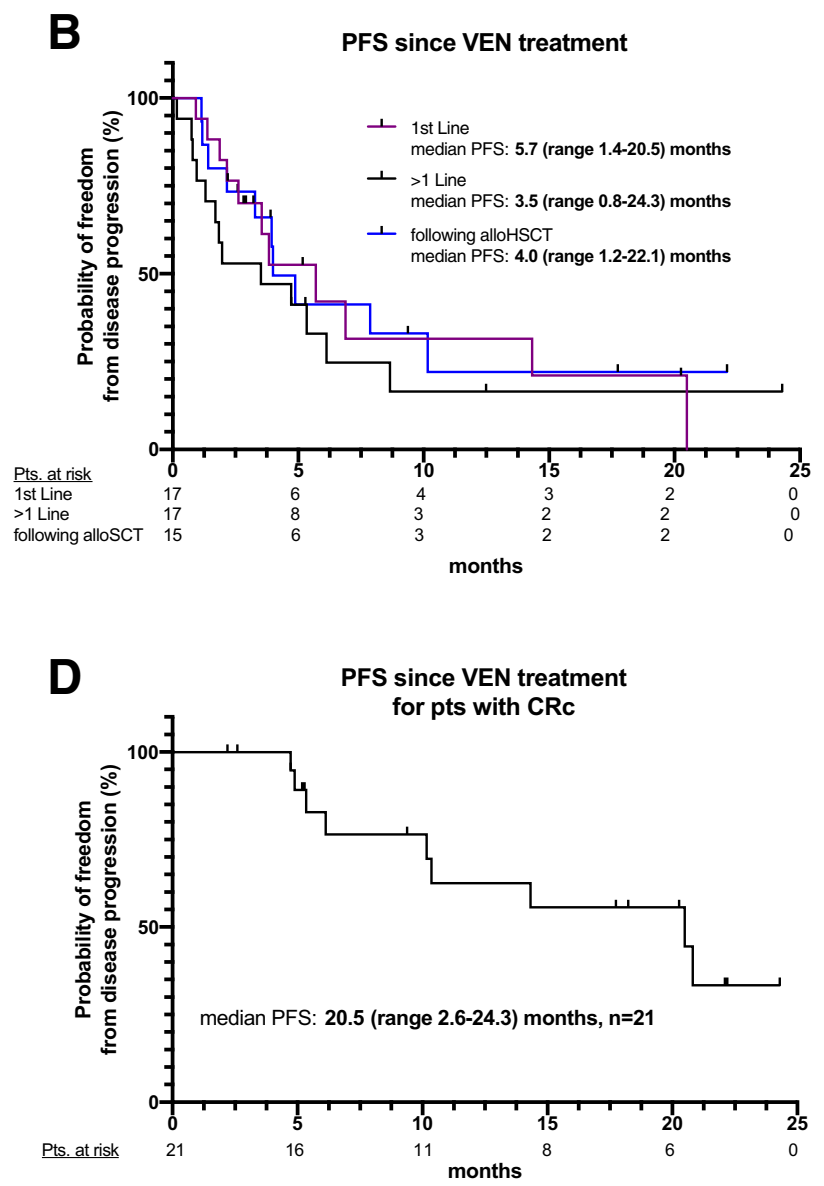

neic hematopoietic stem cell transplantation, $P F S$ progression-free survival, $C R c$ composite complete remission $(\mathrm{CR}+\mathrm{CRi}+\mathrm{CRp}), V E N$ Venetoclax

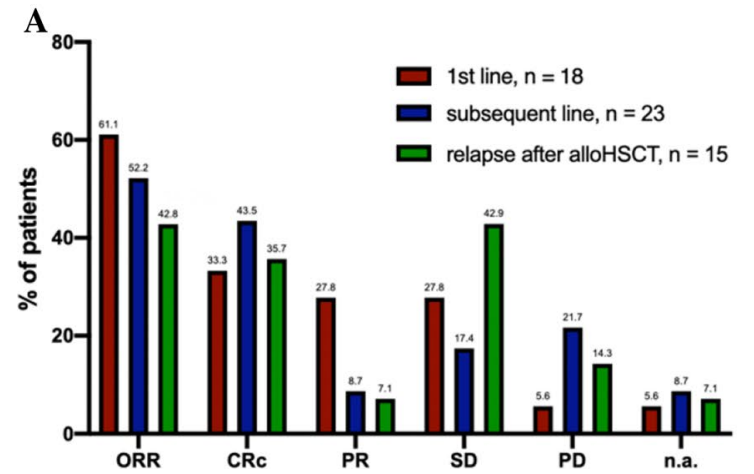

Fig. 3 Bone marrow response rates for treatment cohorts (A) and distinct genetic subgroups (B). alloHSCT allogeneic hematopoietic stem cell transplantation; $O R R$ overall response rate, $P D$ progressive dis-

with an ORR of $12.5 \%$, in contrast $71.4 \%$ in NPM1 and / or IDH1,2 mutated AML, respectively (Fig. 3B). In multivariable analysis, NPM1 mutation retained independent favorable

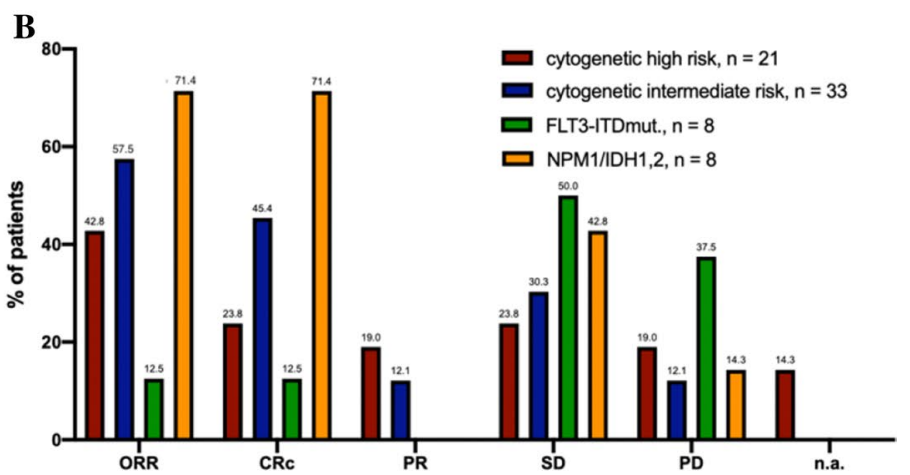

ease, $S D$ stable disease, $P R$ partial remission, $C R c$ composite complete remission $(\mathrm{CR}+\mathrm{CRi}+\mathrm{CRp})$, n.a. not assessed

prognostic significance with regard to achieving CRc (HR $19.14,95 \%$ CI $2.303-436.2, p<0.05)$. 
With respect to genetic subgroups, no difference in survival since VEN initiation was seen for cytogenetic (intermediate vs. high risk) and ELN risk groups (favorable + intermediate vs. high-risk) (Fig. 4A, B): 6.4 (2.2-24.3) months for intermediate vs. 4.9 (1.1-20.8) months for high-risk cytogenetics $(p=0.598)$ and $6.4(1.1-22.2)$ months vs. 5.0 (2.2-24.3) months for ELN high-risk vs. favorable + intermediate, respectively ( $p=0.565)$.

Analysis of distinct molecular subgroups revealed that patients harboring FLT3-ITD mutation $(14.3 \%, n=8)$ had a significantly reduced median OS of $3.4(1.9-4.9)$ months compared to $10.4(0.8-24.3)$ months for those without an activating FLT3-ITD mutation (HR 4.45, 95\% CI 0.89-22.12, $p=0.0002$ ).

In contrast, comparing survival of patients with NPMI, $I D H 1$, or IDH2 mutations without co-occurring FLT3-ITD mutations with the remaining cohort, an increased sensitivity to VEN-based therapy has been demonstrated: $11.2(5$ -24.3) months versus $5.0(0.8-22.1)$, respectively (HR 0.53 , $95 \%$ CI $0.23-1.21, p=0.131$ ) (Fig. 4D).
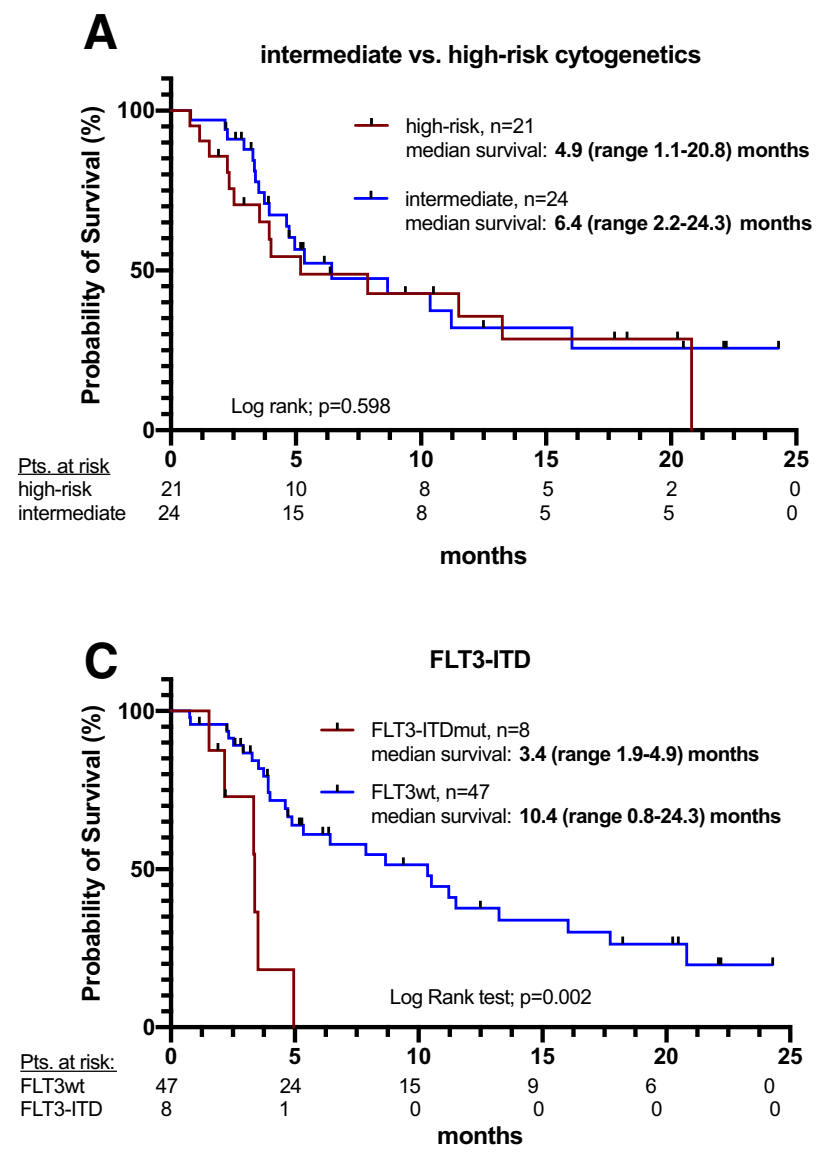

Fig. 4 Kaplan-Meier estimates for survival time were measured from starting VEN treatment for intermediate vs. high-risk cytogenetics (A), ELN 2017 risk groups (B), according to FLT3-ITD (C) and
Furthermore, assessment of blood count and transfusion (in-)dependence was performed. As demonstrated in Table 3, median count for platelets was $12 \times 10^{9} / 1(12-317)$ and $42.5 \times 10^{9} / 1(12-280)$ on days 0 and 100 of treatment, respectively. Transfusion dependence for platelets was decreasing from $62.9 \%$ to $47.2 \%$ and for red blood cells (RBC) from $75.9 \%$ to $55.5 \%$ during the first 100 days of treatment. Median neutrophil count was $0.39 \times 10^{9} / 1$ (range $0.1-12.5$ ) and $0.2 \times 10^{9} / 1$ (range $0.1-3.8$ ) at days 0 and 100 of treatment, respectively. On day 60,17 of 49 patients (36\%) showed an increase of the neutrophil count compared to baseline value.

\section{Response and survival depending on VEN dosage}

The median of the mean VEN dosage of the whole cohort was $149 \mathrm{mg} / \mathrm{d}(20.9-362.5)$ with a median treatment duration of $105(18-674)$ days (Table 2).

To uncover a potential impact of VEN dosing on survival or response rates, patients with a mean daily dose of $\operatorname{VEN} \leq 100 \mathrm{mg}(n=22)$ were compared to those
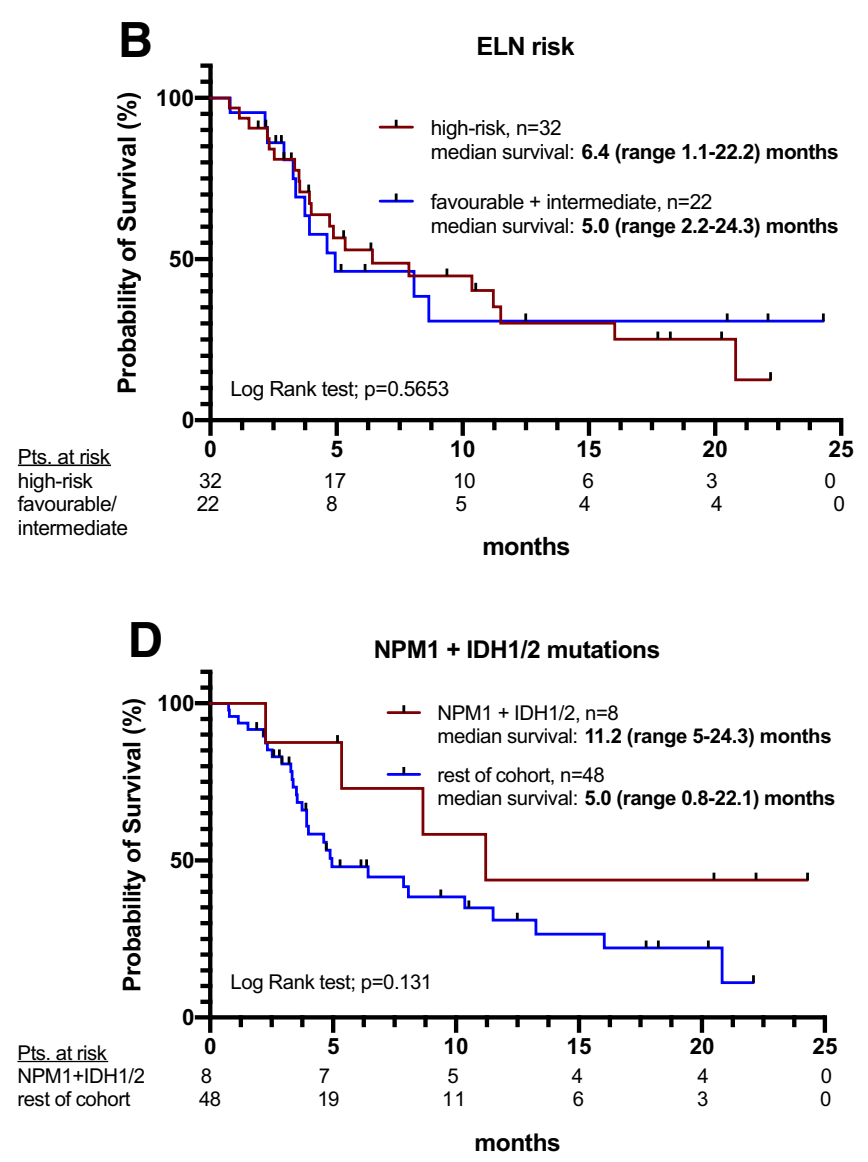

NPM1 or $I D H 1 / 2$ mutational status (D). VEN venetoclax, ELN European leukemia net 
Table 3 Development of blood count and requirement for transfusions under Venetoclax treatment

\begin{tabular}{|c|c|c|c|}
\hline & Platelets d0 (54 pts)* & Platelets d60 (49 pts)* & Platelets d100 (36 pts)* \\
\hline Median count (range) in $10^{9} / 1$ & $12(12-317)$ & $12(12-349)$ & $42.5(12-280)$ \\
\hline Pts with requirement for transfusion & $34(62.9 \%)$ & $27(55.1 \%)$ & $17(47.2 \%)$ \\
\hline \multirow[t]{2}{*}{ Pts lost requirement for transfusion to baseline } & & 10 & +1 \\
\hline & RBC d0 (54 pts) & RBC d60 (49 pts) & RBC d100 (36 pts) \\
\hline Median count (range) in $10^{9} / 1$ & $4.5(4.5-8)$ & $4.5(4.5-7.3)$ & $4.5(4.5-8.3)$ \\
\hline Pts with requirement for transfusion & $41(75.9 \%)$ & $36(73.4 \%)$ & $20(55.5 \%)$ \\
\hline \multirow[t]{2}{*}{ Pts lost requirement for transfusion to baseline } & & 7 & +1 \\
\hline & $\mathrm{ANC}$ d0 (52 pts) & ANC d60 (47 pts) & ANC d100 (34 pts) \\
\hline Median count (range) in $10^{9} / 1$ & $0.39(0.1-12.5)$ & $0.2(0.1-4.3)$ & $0.2(0.1-3.8)$ \\
\hline \multicolumn{2}{|c|}{ Pts with increase of neutrophils compared to baseline } & 17 & +3 \\
\hline
\end{tabular}

pts patients; $d$ days; $A N C$ absolute neutrophils count; $R B C$ red blood count

*Number of patients on treatment

with $>100 \mathrm{mg}(\mathrm{n}=34)$. Kaplan-Meier analysis demonstrates a median survival of 6.4 (1.5-17.7) months and 8.1 (1.1-24.3) months for patients having received a mean daily dose of $\leq 100 \mathrm{mg}$ and $>100 \mathrm{mg}$, respectively $(\mathrm{p}=0.357)$ (Fig. 5A). Doses of $>100 \mathrm{mg}$ showed an increased ORR $(55.9 \%)$ compared with patients receiving $\leq 100 \mathrm{mg}$ mean dosage $(45.5 \%)$. Progressive disease while on VEN treatment was noted in $22.3 \%$ and $8.9 \%$ comparing the cohorts of $\leq 100 \mathrm{mg}$ and $>100 \mathrm{mg}$, respectively (Fig. 5B).

\section{Toxicity assessment}

Most frequent grade 3 and 4 side effects were hematologic with neutropenia, thrombocytopenia, and anemia (44.6\%, 14.5\%, 12\%, respectively) (Fig. 6). Grade 3 renal insufficiency occurred in 3 patients $(5.3 \%)$ including two cases of tumor lysis syndrome. One patient suffered from severe retinal bleeding, another patient from dysesthesia und body aches. Common adverse events are summarized in Fig. 6. Non-relapse mortality rate was $8.9 \%$ (5/56 patients) and early deaths till day 30 since treatment start occurred in $3.5 \%$ (2/56 patients).

\section{Discussion}

The current study reports on 56 treatment naïve and $\mathrm{r} / \mathrm{r}$ adult AML patients treated with VEN combination therapy at an academic site, outside clinical trials. Detailed analyses with respect to different disease settings were performed:

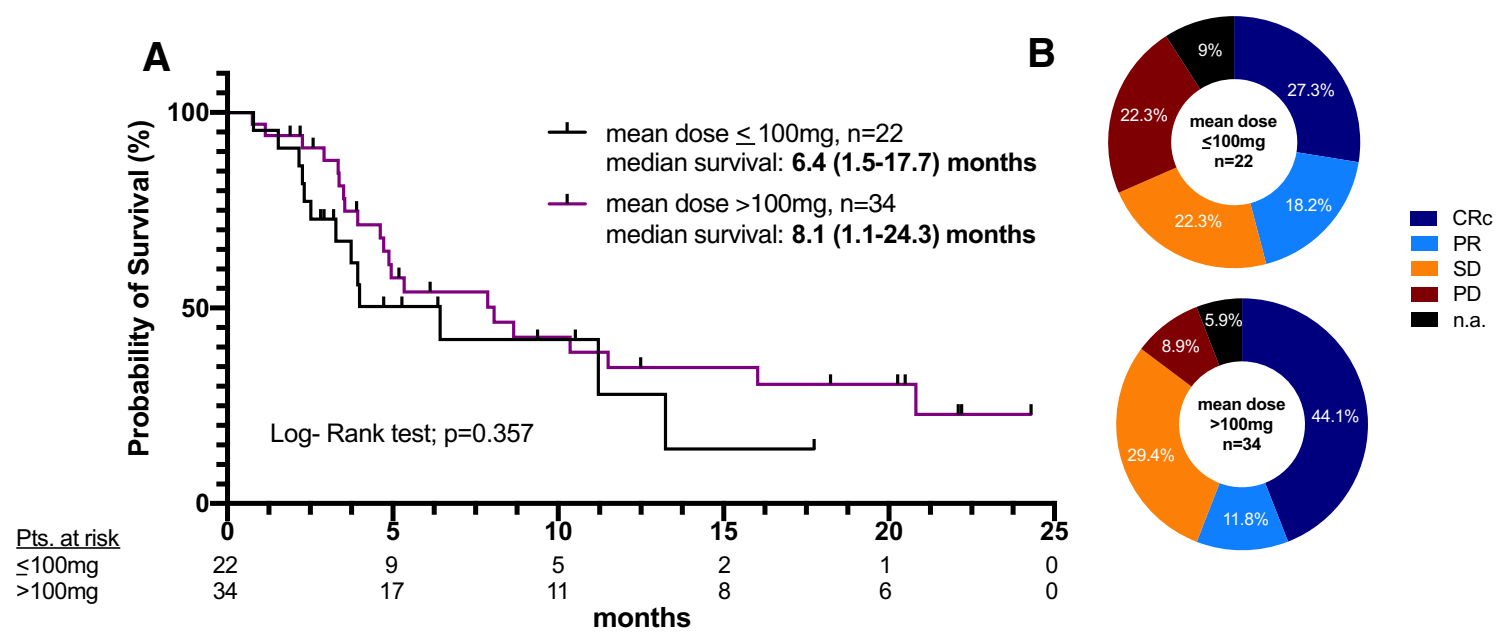

Fig. 5 Kaplan-Meier estimates for survival time since start of VEN treatment according to mean dosage of VEN (A). Bone marrow response rates according to mean dosage of VEN (B). CRc compos- ite complete remission $(\mathrm{CR}+\mathrm{CRi}+\mathrm{CRp}), P R$ partial remission, $S D$ stable disease, $P D$ progressive disease, n.a. not assessed, VEN venetoclax 
Fig. 6 Assessment of VEN toxicities according to CTC classification. $V E N$ venetoclax, CTC common toxicity criteria

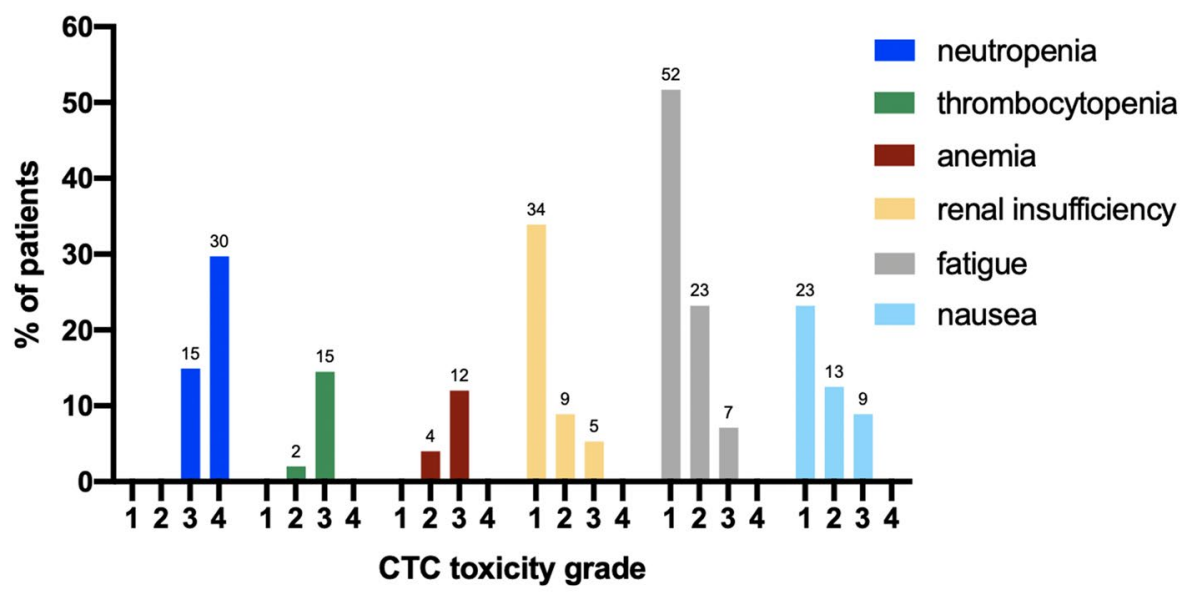

VEN as first-line, subsequent line (r/r AML excluding prior alloHSCT) and VEN at relapse post-alloHSCT.

Introduction of VEN combination regimens in first-line treatment has improved survival rates in AML patients not eligible for intensive chemotherapy (DiNardo et al. 2020; Wei et al. 2020). DiNardo et al. report a median OS of 14.7 months in the first-line AZA-VEN group (286 patients) and $9.6(7.4-12.7)$ months in the AZA control group (HR $0.66,95 \%$ CI $0.52-0.85, p<0.001$ ) (DiNardo et al. 2020). In our cohort, a median OS of 13.3 (2.2 - 20.5) months for patients who were treated as first-line therapy with VEN was achieved. Furthermore, survival data are comparable to other published reports outside clinical trials. Recently, a study by Apel et al. evaluated 103 newly diagnosed AML patients and could demonstrate an OS of 9.8 (6.4-13.3) months (Apel et al. 2021).

As expected, differences in survival rates between subgroups were noticed. When applied as subsequent therapy ( $\mathrm{r} / \mathrm{r}$ AML excluding prior alloHSCT), median OS was 5.0 $(0.8-24.3)$ months and in case of relapse post-alloHSCT $4.0(1.5-22.1)$ months, respectively.

Clinical scenarios, such as failure of conventional induction chemotherapy or relapse post-alloHSCT, remain a challenge. Especially in genetically high-risk patients, re-induction by conventional chemotherapy is associated with poor response rates (Roboz et al. 2014; Mangan and Luger 2011). The use of VEN combination therapy might overcome the adverse prognosis of distinct cytogenetic and molecular aberrations. Within a small subgroup of 11 patients, VEN was applied as salvage therapy after induction failure and 5 patients were transitioned to alloHSCT. A high ORR rate of $54.5 \%$ was achieved in those 11 patients, thus exceeding ORR rates of chemotherapy-based salvage regimens in patients harboring adverse genetics (Ravandi et al. 2010). Recently, a salvage regimen combining VEN and FLAGIDA has been demonstrated convincing response rates in $\mathrm{r} / \mathrm{r}$
AML (CRc rate of 76\%) providing a promising approach for this challenging patient cohort (DiNardo et al. 2021).

Another major therapeutic challenge with a high unmet need remains post-alloHSCT relapse. Relapse after alloHSCT occurs in almost half of AML patients and survival remains dismal (Schmid et al. 2018; Barrett and Battiwalla 2010). Treatment with HMAs in this setting resulted in an ORR of $19 \%$ and a low CR rate of $7 \%$, whereas the utility of VEN in post-transplant settings is poorly studied (Motabi et al. 2016). 15 out of 56 patients of our cohort received VEN combination at relapse post-alloHSCT. Within this subgroup, 36\% achieved CRc and another 7\% PR. This is of importance since many of post-alloHSCT r/r AML patients are not eligible for further intensive salvage treatment. A retrospective analysis of 20 post-alloHSCT relapse patients showed a CRc rate of $70 \%$ when treated with a combination therapy consisting of VEN, LDAC and Actinomycin D (Zucenka et al. 2021). Another recently published retrospective study on r/r AML patients after alloHSCT including 29 patients demonstrated an ORR of $38 \%$ with a median OS of 2.6 months (Joshi et al. 2021). Taken together, VEN combination strategies represent a rational salvage strategy in post-alloHSCT r/r AML patients with encouraging CR rates that might offer the chance of another subsequent potential curative alloHSCT.

In palliative $\mathrm{r} / \mathrm{r}$ AML patients not eligible for intensive treatment even at initial diagnosis who received VEN as subsequent line therapy, response rates and survival were considerably reduced compared to first-line therapy. Nevertheless, $43 \%$ of those r/r AML patients within our cohort attained CRc suggesting a reasonable therapeutic approach in this difficult-to-treat patient cohort. Comparable data of response rates have been reported on $\mathrm{r} / \mathrm{r}$ AML patients on VEN treatment with a CR rate of $32 \%$ and a median OS of 5.5 months compared to 5.0 months in our cohort (Tenold et al. 2021). Treatment options in elderly patients at AML relapse are limited. Gilteritinib plays a role in FLT3-mutated 
AML as it is approved in the $\mathrm{r} / \mathrm{r}$ setting (Perl et al. 2019). However, the frequency of FLT3 mutations accounts for about $25 \%$ of AML patients, so a majority of patients do not benefit from the FLT3-inhibitor. HMAs resulted in a CR rate of $16 \%$ in $\mathrm{r} / \mathrm{r}$ AML as reported in a large international patient cohort (Stahl et al. 2018). Considering the limited treatment options, our data underline the benefit of VEN-based therapy in elderly and frail $\mathrm{r} / \mathrm{r}$ AML patients.

Across genetic risk groups, no difference in survival was observed when stratified by cytogenetics and ELN criteria. Detailed molecular genetic analyses revealed a significantly worse outcome in FLT3-ITD-mutated. Median OS from VEN to follow-up was $3.4(1.4-4.9)$ months in FLT3-ITDmutated versus 10.4 (0.8 - 24.3) months in FLT3-wildtype patients, respectively.

Conflicting data exist on the impact of FLT3-ITDmutated AML and VEN activity. Pre-clinical models suggest that FLT3-ITD activation mediates resistance to VEN (Singh Mali et al. 2021). STAT5 activation by FLT3-ITD leads to regulation of pro-survival proteins $\mathrm{BCL}(\mathrm{x}) \mathrm{L}$ and MCL-1. These molecules are known for conferring VEN resistance; thus, combining BCL-2 inhibition and FLT3ITD blockade might be a therapeutic rationale (Zhu et al. 2021; Maiti et al. 2021). On the other hand, DiNardo and colleagues reported a CR rate of $72 \%$ in newly diagnosed FLT3-ITD-mutated AML patients $(n=10)$ (DiNardo et al. 2020). Noteworthy, those data are based on limited patient numbers or preclinical models. Larger data sets are needed for a conclusive statement especially considering variables like allele burden of FLT3-ITD and concurrent mutations.

NPM1-, IDH1-, or IDH2-mutated AML (and FLT3-WT) had favorable responses to VEN which have also been documented by others (DiNardo et al. 2021). However, cautious interpretation of these molecular subgroup analyses is warranted due to the small sample size and exploratory nature.

In line with the response data, a relevant decrease in transfusion frequency could be achieved. At VEN initiation, transfusion of platelets and RBCs was necessary in $63 \%$ and $76 \%$ of patients, respectively. At day 100 of VEN treatment, $47 \%$ required platelet and 55\% RBC transfusions, respectively.

When evaluating subsequent treatment strategies after VEN exposure, responses were only seen in patients undergoing alloHSCT, indicating an urgent medical need for patients progressing/relapsing on VEN treatment.

As expected, cytopenia is a major concern in VEN treatment. Particularly, $45 \%$ of patients experienced grade 4 neutropenia. Several expert opinions have been proposed to manage those commons side effects including G-CSF application, shortening of VEN treatment, reduction of VEN or HMA dose, respectively (Jonas and Pollyea 2019; Winters et al. 2019; Rausch et al. 2021). In our patient cohort treatment, interruption was performed in $29 \%$ of patients and nearly exclusively due to neutropenia. Importantly, co-administration of CYP3A4 inhibiting azoles should be addressed with a dose reduction of VEN since CYP3A4 is the primary enzyme responsible for the metabolism of VEN (Rausch et al. 2021).

By comparing probability of survival in patients who received a mean dose of $100 \mathrm{mg}$ or less (22 patients) compared to doses higher than $100 \mathrm{mg}$ (34 patients), no significant difference was seen with a median survival since VEN treatment of 6.4 (1.5-17.7) months or 8.1 (1.1-24.3) months, respectively. Although not statistically significant, patients treated with higher doses of VEN had a greater likelihood of achieving a CRc (44\% versus 27\%). Measuring individual VEN plasma concentrations and considering concurrent azole medication would yield a much more reliable result for exposure-efficacy relationship. Furthermore, genetic heterogeneity of AML, such as upregulation of BCL2AI and CLEC7A or mutations of PTPNII and KRAS, confers resistance to VEN, which has not been considered (Zhang et al. 2020).

In conclusion, VEN treatment shows impressive response rates when applied as first-line treatment suggesting VENbased combination approaches also for curative intended AML patients either characterized by relevant comorbidities or harboring unfavorable cytogenetic risk factors associated with a high rate of induction failure. Furthermore, in $\mathrm{r} / \mathrm{r}$ AML including relapse after alloHSCT, it represents a reasonable therapeutic approach. Those who fail on VEN treatment and are not eligible for intensive therapy have a poor prognosis and alternative treatment strategies are required.

Supplementary Information The online version contains supplementary material available at https://doi.org/10.1007/s00432-022-03930-5.

Funding Open Access funding enabled and organized by Projekt DEAL. No funding.

Availability of data and material Patient records of University Hospital Jena.

\section{Declarations}

Conflict of interest The authors declare no conflict of interest.

Ethical approval Data analysis has been approved by local ethics committee (3967-12/13 for SAL registry).

Consent to participate Informed consent of patients for participation was obtained when registered for SAL registry (see above).

Consent for publication Consent of patients to publish was obtained when registered for SAL registry (see above).

Open Access This article is licensed under a Creative Commons Attribution 4.0 International License, which permits use, sharing, adaptation, distribution and reproduction in any medium or format, as long 
as you give appropriate credit to the original author(s) and the source, provide a link to the Creative Commons licence, and indicate if changes were made. The images or other third party material in this article are included in the article's Creative Commons licence, unless indicated otherwise in a credit line to the material. If material is not included in the article's Creative Commons licence and your intended use is not permitted by statutory regulation or exceeds the permitted use, you will need to obtain permission directly from the copyright holder. To view a copy of this licence, visit http://creativecommons.org/licenses/by/4.0/.

\section{References}

Aldoss I, Yang D, Aribi A et al (2018) Efficacy of the combination of venetoclax and hypomethylating agents in relapsed/refractory acute myeloid leukemia. Haematologica 103:e404-e407

Apel A, Moshe Y, Ofran Y et al (2021) Venetoclax combinations induce high response rates in newly diagnosed acute myeloid leukemia patients ineligible for intensive chemotherapy in routine practice. Am J Hematol 96:790-795

Barrett AJ, Battiwalla M (2010) Relapse after allogeneic stem cell transplantation. Expert Rev Hematol 3:429-441

Beelen DW, Trenschel R, Stelljes M et al (2020) Treosulfan or busulfan plus fludarabine as conditioning treatment before allogeneic haemopoietic stem cell transplantation for older patients with acute myeloid leukaemia or myelodysplastic syndrome (MCFludT.14/L): a randomised, non-inferiority, phase 3 trial. Lancet Haematol 7:e28-e39

Casper J, Holowiecki J, Trenschel R et al (2012) Allogeneic hematopoietic SCT in patients with AML following treosulfan/fludarabine conditioning. Bone Marrow Transplant 47:1171-1177

DiNardo CD, Rausch CR, Benton C et al (2018) Clinical experience with the BCL2-inhibitor venetoclax in combination therapy for relapsed and refractory acute myeloid leukemia and related myeloid malignancies. Am J Hematol 93:401-407

DiNardo CD, Jonas BA, Pullarkat V et al (2020) Azacitidine and venetoclax in previously untreated acute myeloid leukemia. $\mathrm{N}$ Engl J Med 383:617-629

DiNardo CD, Lachowiez CA, Takahashi K et al (2021) Venetoclax combined with FLAG-IDA induction and consolidation in newly diagnosed and relapsed or refractory acute myeloid leukemia. J Clin Oncol 39:2768-2778

Döhner H, Estey E, Grimwade D et al (2017) Diagnosis and management of AML in adults: 2017 ELN recommendations from an international expert panel. Blood 129:424-447

Dombret H, Seymour JF, Butrym A et al (2015) International phase 3 study of azacitidine vs conventional care regimens in older patients with newly diagnosed AML with $>30 \%$ blasts. Blood 126:291-299

Gale RP, Barosi G, Barbui T et al (2011) What are RBC-transfusiondependence and -independence? Leuk Res 35:8-11

Jethava YS, Sica S, Savani B et al (2017) Conditioning regimens for allogeneic hematopoietic stem cell transplants in acute myeloid leukemia. Bone Marrow Transplant 52:1504-1511

Jonas BA, Pollyea DA (2019) How we use venetoclax with hypomethylating agents for the treatment of newly diagnosed patients with acute myeloid leukemia. Leukemia 33:2795-2804

Joshi M, Cook J, McCullough K et al (2021) Salvage use of venetoclax-based therapy for relapsed AML post allogeneic hematopoietic cell transplantation. Blood Cancer J 11:49

Juliusson G, Antunovic P, Derolf A et al (2009) Age and acute myeloid leukemia: real world data on decision to treat and outcomes from the Swedish acute leukemia registry. Blood 113:4179-4187
Kantarjian HM, Thomas XG, Dmoszynska A et al (2012) Multicenter, randomized, open-label, phase III trial of decitabine versus patient choice, with physician advice, of either supportive care or lowdose cytarabine for the treatment of older patients with newly diagnosed acute myeloid leukemia. J Clin Oncol 30:2670-2677

Kayser S, Levis MJ (2021) Updates on targeted therapies for acute myeloid leukaemia. Br J Haematol. https://doi.org/10.1111/bjh. 17746

Maiti A, DiNardo CD, Qiao W et al (2021) Ten-day decitabine with venetoclax versus intensive chemotherapy in relapsed or refractory acute myeloid leukemia: a propensity score-matched analysis. Cancer. https://doi.org/10.1016/S2152-2650(21)01362-8

Maiti A, DiNardo CD, Daver NG et al (2021) Triplet therapy with venetoclax, FLT3 inhibitor and decitabine for FLT3-mutated acute myeloid leukemia. Blood Cancer J 11:25

Mangan JK, Luger SM (2011) Salvage therapy for relapsed or refractory acute myeloid leukemia. Ther Adv Hematol 2:73-82

McGowan-Jordan J, Hastings R, Moore S (2021) Re: International system for human cytogenetic or cytogenomic nomenclature (ISCN): some thoughts, by T. Liehr. Cytogenet Genome Res $161: 225-226$

Motabi IH, Ghobadi A, Liu J et al (2016) Chemotherapy versus hypomethylating agents for the treatment of relapsed acute myeloid leukemia and myelodysplastic syndrome after allogeneic stem cell transplant. Biol Blood Marrow Transplant 22:1324-1329

Perl AE, Martinelli G, Cortes JE et al (2019) Gilteritinib or chemotherapy for relapsed or refractory FLT3-mutated AML. N Engl J Med 381:1728-1740

Piccini M, Pilerci S, Merlini M et al (2021) Venetoclax-based regimens for relapsed/refractory acute myeloid leukemia in a real-life setting: a retrospective single-center experience. J Clin Med. https:// doi.org/10.3390/jcm10081684

Pollyea DA, Amaya M, Strati P, Konopleva MY (2019) Venetoclax for AML: changing the treatment paradigm. Blood Adv 3:4326-4335

Rausch CR, DiNardo CD, Maiti A et al (2021) Duration of cytopenias with concomitant venetoclax and azole antifungals in acute myeloid leukemia. Cancer 127:2489-2499

Ravandi F, Cortes J, Faderl S et al (2010) Characteristics and outcome of patients with acute myeloid leukemia refractory to 1 cycle of high-dose cytarabine-based induction chemotherapy. Blood 116:5818-5823 (quiz 6153)

Roboz GJ, Rosenblat T, Arellano M et al (2014) International randomized phase III study of elacytarabine versus investigator choice in patients with relapsed/refractory acute myeloid leukemia. J Clin Oncol 32:1919-1926

Samra B, Konopleva M, Isidori A et al (2020) Venetoclax-based combinations in acute myeloid leukemia: current evidence and future directions. Front Oncol 10:562558

Schmid C, de Wreede LC, van Biezen A et al (2018) Outcome after relapse of myelodysplastic syndrome and secondary acute myeloid leukemia following allogeneic stem cell transplantation: a retrospective registry analysis on 698 patients by the chronic malignancies working party of the European society of blood and marrow transplantation. Haematologica 103:237-245

Singh Mali R, Zhang Q, DeFilippis RA et al (2021) Venetoclax combines synergistically with FLT3 inhibition to effectively target leukemic cells in FLT3-ITD+ acute myeloid leukemia models. Haematologica 106:1034-1046

Stahl M, DeVeaux M, Montesinos P et al (2018) Hypomethylating agents in relapsed and refractory AML: outcomes and their predictors in a large international patient cohort. Blood Adv 2:923-932

Stasik S, Middeke JM, Kramer M et al (2020) EZH2 mutations and impact on clinical outcome: an analysis in 1604 patients with newly diagnosed acute myeloid leukemia. Haematologica 105:e228-e231 
Tenold ME, Moskoff BN, Benjamin DJ et al (2021) Outcomes of adults with relapsed/refractory acute myeloid leukemia treated with venetoclax plus hypomethylating agents at a comprehensive cancer center. Front Oncol 11:649209

Wei AH, Montesinos P, Ivanov V et al (2020) Venetoclax plus LDAC for newly diagnosed AML ineligible for intensive chemotherapy: a phase 3 randomized placebo-controlled trial. Blood 135:2137-2145

Winters AC, Gutman JA, Purev E et al (2019) Real-world experience of venetoclax with azacitidine for untreated patients with acute myeloid leukemia. Blood Adv 3:2911-2919

Zhang H, Nakauchi Y, Köhnke T et al (2020) Integrated analysis of patient samples identifies biomarkers for venetoclax efficacy and combination strategies in acute myeloid leukemia. Nat Cancer $1: 826-839$
Zhu R, Li L, Nguyen B et al (2021) FLT3 tyrosine kinase inhibitors synergize with BCL-2 inhibition to eliminate FLT3/ITD acute leukemia cells through BIM activation. Signal Transduct Target Ther 6:186

Zucenka A, Vaitekenaite V, Maneikis K et al (2021) Venetoclax-based salvage therapy followed by venetoclax and DLI maintenance vs. FLAG-Ida for relapsed or refractory acute myeloid leukemia after allogeneic stem cell transplantation. Bone Marrow Transplant. https://doi.org/10.1038/s41409-021-01416-5

Publisher's Note Springer Nature remains neutral with regard to jurisdictional claims in published maps and institutional affiliations. 